\title{
Factores de riesgo asociados a la violencia sufrida por la mujer en la pareja: una revisión de meta-análisis y estudios recientes
}

\author{
Alicia Puente-Martínez ${ }^{*}$, Silvia Ubillos-Landa ${ }^{2}$, Enrique Echeburúa ${ }^{1}$ y Darío Páez-Rovira ${ }^{1}$ \\ ${ }^{1}$ Universidad del País Vasco UPV-EHU (España). \\ ${ }^{2}$ Universidad de Burgos UBU (España).
}

\begin{abstract}
Resumen: El objetivo de este trabajo fue realizar una revisión bibliográfica actual y complementaria de los últimos estudios y meta-análisis sobre factores de riesgo de la violencia de género. Este trabajo confirma que en el nivel socio-comunitario el bajo nivel educativo, el bajo desarrollo económico del país, pocos derechos sociales, la falta de democratización del Estado, la cultura del honor y las culturas masculinas - con actitudes sexistas y favorables hacia la violencia en el país- son factores de riesgo. En el nivel contextual e individual, tener bajos ingresos, tener menor edad, más hijos, la violencia recíproca hacia la pareja, la depresión, el miedo y el consumo de alcohol, se asocian a mayor riesgo de ser víctima de violencia por parte de la pareja íntima. Con menor consistencia, son factores de riesgo, las situaciones de guerra, el fundamentalismo religioso, la mayor duración de la relación y una menor satisfacción con la pareja, así como emociones como la culpa, vergüenza y otros factores como el embarazo.
\end{abstract}

Palabras clave: Violencia de género; victima; agresor; factor de riesgo.
Title: Risk factors associated with the violence against women in couples: a review of meta-analyzes and recent studies.

Abstract: The aim of this study was to conduct a complementary to current and recent meta-analysis of risk factors to intimate partner violence literature review. This work confirms that on community-level, low economic development and democracy, lack of social rights, culture of honor and masculine culture - characterized by sexist attitudes and tolerance to violence- are risk factors. On contextual and individual level, being younger, having a low income and low education level, having more than one child, using violence reciprocally against ones partner, depression, fear and alcohol consumption are associated with increased risk of being a victim of intimate violence. Less consistency, are risk factors, situations of war, religious fundamentalism, being in a long term relationship, lower relationship satisfaction, emotions such as guilt, shame and other factors such as pregnancy.

Key words: Gender violence; victim; aggressor; risk factor.

\section{Introducción}

La violencia por parte de la pareja (VPI) es una lacra social y un fenómeno frecuente con importantes repercusiones sobre la salud de las víctimas y con un fuerte impacto social. La VPI se define como una forma de violencia que surge dentro de las relaciones íntimas y de pareja actuales o pasadas, ejercida por los hombres sobre las mujeres por su condición de género y que incluye conductas de abuso como son el maltrato físico, psicológico, sexual y otros comportamientos de tipo controlador, intimidación o las amenazas coercitivas (Adelman, 2003). Un estudio realizado en distintos países ( $n=24.000$ mujeres) por la OMS (2005) estima que entre el 15\% en Japón hasta el $79 \%$ en Perú de las mujeres con edades comprendidas entre 15 y 49 años han sufrido violencia física y/o sexual por parte de su pareja en algún momento de su vida. Se calcula que en la infancia un $20 \%$ de mujeres ha sido víctima de violencia frente a un $10 \%$ de hombres. En España, el porcentaje de mujeres maltratadas asciende al 9,6\%, del cual la mayor parte tienen entre los 45 y 64 años (12\%) (Instituto de la Mujer, 2007).

La violencia sufrida por la mujer en la pareja se manifiesta en diferentes niveles. Las expresiones de la conducta violenta en la pareja tienen un origen multicausal y existen factores de riesgo específicos muy diversos asociados a la violencia (Pueyo y Redondo, 2007). Numerosas teorías dan cuenta de la aparición y del mantenimiento de la violencia en la pareja. Dutton (1995) propone un modelo ecológico, donde explica que las variables que contribuyen a la violen-

* Dirección para correspondencia [Correspondence address]: Alicia Puente Martínez. Universidad del País Vasco/Euskal Herrico Unibersitatea. Avenida Tolosa, 70. CP 2018. San Sebastián (España). E-mail: alicia.puente@,ehu.es cia se sitúan en varios niveles: macroestructural o social; exosistema (regional); nivel micro (grupal) y ontogenético (individual). Este modelo proporciona un marco teórico dentro del cual se consideran las relaciones específicas entre los predictores de la violencia y de la agresión en la pareja. De este modo, de lo macro a lo micro hay un continuo que va de lo social a lo individual. Ninguno de los niveles puede considerarse fundamental o prioritario, sino que los distintos sistemas se implican entre sí.

De esto se deduce, que las características de cada país influyen en el alcance de la violencia en general y en la pareja en particular. Desde la macro-estructura influyen los niveles de globalización, el ámbito político, económico y los aspectos culturales. En el exosistema se incluyen las estructuras individuales formales e informales. En el nivel micro se sitúan los grupos o unidades de interacción que influyen en la actualización y transmisión de la violencia - los que hacen referencia al contexto de la relación, al nivel de satisfacción y a la armonía familiar. Por último, en el nivel ontogenético se integran las características individuales y referidas a la historia de abusos. Se dan distintas explicaciones que hacen referencia a alguno de los niveles: las teorías feministas enfatizan lo societal, es decir la estructura patriarcal, o el papel de las actitudes y los roles sexuales en la configuración de las relaciones de poder y de abuso a la pareja, centrándose en los procesos de socialización facilitadores e inhibidores como la familia, escuela y medios de comunicación (Pérez, Bosch, Navarro y Ramis, 2006); los sociólogos se centran en el análisis del contexto social como determinante de la conducta; las teorías psicológicas en las diferencias individuales en víctimas y agresores basadas en las características psicológicas y rasgos de personalidad y los del aprendizaje explican la agresión a partir de la exposición a modelos violentos, subculturas violentas, confrontaciones o situaciones de crisis sociales 
intensas (Echeburúa, Sarasua, Zubizarreta, Amor y Corral, 2010). Otros autores ponen énfasis en la interacción de los procesos individuales y sociales. Los atributos psicológicos individuales pueden funcionar como variables predisponentes que actúan en combinación con determinados factores sociales como variables desencadenantes (Pueyo y Redondo, 2007).

Debido a la multifactorialidad de la violencia de género, examinar cuáles son los factores de riesgo de este tipo de comportamientos es una tarea difícil. Por eso, además de analizar estudios independientes recientes que evaluaron los riesgos de sufrir abuso por parte de la pareja, se estudian las relaciones entre estos factores. El número de factores de riesgo importantes reflejado en esta revisión demuestra la complejidad en la comprensión de las causas y correlaciones de la violencia sufrida por parte de la pareja.

El objetivo de este trabajo es realizar una revisión bibliográfica actual y estructurada basada en los resultados encontrados en los últimos estudios y meta-análisis sobre los factores de riesgo que tradicionalmente se han asociado con un incremento de violencia de género.

\section{Revisión de los meta-análisis}

El primer meta-análisis de Hotaling y Sugarman (1986) basado en más de 50 estudios agrupa aquellos indicadores que se han asociado al incremento de la violencia por parte de la pareja. Estos autores establecen cuatro categorías independientes: a) factores consistentes o significativos que aparecen en al menos tres investigaciones independientes (del total de los artículos revisados el factor de riesgo estaba presente en el $70 \%$ de los casos y se tuvo en cuenta para su inclusión en el estudio), como son el ser testigo de violencia en la infancia; b) inconsistentes o en los que no existe un patrón de consistencia a lo largo de los diferentes estudios (entre el 3169\% de los casos analizados), como son: experiencias de violencia en la infancia, abuso de drogas, autoestima, nivel educativo, roles sexuales tradicionales, sexo, edad, raza, dominancia, primer matrimonio y hostilidad; c) factores de riesgo no consistentes o presentes en el 30\% de los estudios: estatus social, el alcohol y los ingresos y, d) con datos insuficientes (presentes en menos de tres investigaciones) como: religión, ocupación, pasividad y la fuerza del ego. Un segundo meta-análisis encontró una relación entre deseabilidad social y subdeclaración de violencia de género en hombres y mujeres (Sugarman y Hotaling, 1997). Otra revisión con 33 estudios obtuvo una correlación positiva entre las actitudes positivas hacia el uso de la violencia y la violencia perpetrada, así como, entre las actitudes tradicionales sobre los roles de género de las mujeres y la perpetración de actos violentos ( $\mathrm{Su}$ garman y Frankel, 1996). Dos meta-análisis encuentran una relación entre la violencia doméstica y presenciar o experimentar violencia familiar en la infancia (Riggs, Culfield y Street, 2000; Stith, Rosen, Middleton, Busch, Lundeberg y Carlton, 2000). Ochenta y dos estudios independientes encontraron que dentro de las relaciones familiares y de pareja, las mujeres son más propensas que los hombres a ejercer algunas formas de agresión menos graves contra su pareja aunque las mujeres tienen una probabilidad mayor de sufrir lesiones graves como heridas y golpes (Archer, 2000). Las experiencias anteriores de violencia como ser testigo de violencia, el abuso de sustancias y la psicopatología previa aumentan el riesgo de ser víctima de violencia en la edad adulta (Riggs et al., 2000; Schumacher, Feldbau-Kohn, Smith y Heyman, 2001).

El meta-análisis de Stith, Smith, Penn, Ward y Tritt (2004) analiza la violencia en la pareja desde la perspectiva socio-ecológica y calcula el tamaño del efecto global para distintos factores de riesgo. Bajo este mismo modelo otra revisión meta-analítica analiza los factores que justifican la violencia en la pareja. Encuentra que una mejor situación económica, mayor edad, más tasa de alfabetización de los hombres, mejor índice de desarrollo de género y de desarrollo humano disminuyen las actitudes de justificación de la violencia (Uthman, Lawoko y Moradi, 2010).

Por último, otros meta-análisis posteriores analizan las consecuencias de la violencia sobre la salud de las víctimas (Beydoun, Beydoun, Kaufman y Zonderman, 2012) o la relación entre las actitudes a favor de la pornografía y la violencia contra la pareja (Suárez y Gadalla, 2010).

\section{Método}

Con el objetivo de recopilar estudios recientes que pudieran dar cuenta de la heterogeneidad de los resultados, se incluyeron aquellos estudios en los que: (a) se mencionan los factores de riesgo que incrementaban la probabilidad de sufrir VPI; (b) se aplican cuestionarios estandarizados de fiabilidad probada reconocidos internacionalmente; (c) son publicados entre los años 2000-2013; y además (d) incluyen datos cuantitativos necesarios para el cálculo de al menos un tamaño del efecto.

Sobre la base anterior, como procedimientos de búsque$\mathrm{da}$, se utilizaron fuentes formales e informales. En primer lugar, se han realizado búsquedas computarizadas en las bases de datos Web, WOS y PsycInfo, SciELO y Pubmed, seleccionando las publicaciones tanto en inglés como en castellano. Como criterios para la búsqueda, se utilizan los factores de riesgo mencionados en el meta-análisis de Stith et al. (2004). Estos factores se han demostrado capaces de predecir un incremento sobre la VPI y a partir de ellos se impulsó la búsqueda de estudios más recientes. La estructura propuesta desde el modelo socio-ecológico, adoptada por este autor, permitió realizar la búsqueda detallada de cada uno de los factores de riesgo hasta el momento y organizar de forma coherente cada uno de los factores encontrados en niveles de agrupación que van de lo comunitario contextual a lo más individual.

Los resúmenes se leyeron y seleccionaron de forma independiente. Las versiones completas de los artículos eran obtenidas si el resumen cumplía los criterios de inclusión a y b, c y d. En segundo lugar, se escribió a investigadores de 
prestigio y expertos en la materia solicitándoles el envío de trabajos o información detallada sobre algunos estudios. Finalmente se revisaron las referencias de las investigaciones recuperadas.

Inicialmente, las cadenas de búsqueda en las bases en inglés se han realizado mediante la combinación sistemática de los siguientes términos en relación a la VPI en los abstract o resúmenes de cada artículo: intimate partner violence and risk. factor, intimate partner violence and abuse; spousal/spouse and violence, couple and violence, couple and victim, couple and aggression, risk. factor and meta-analysis and violence against women. Este mismo criterio se siguió para realizar la búsqueda en español. Además se incluyeron los términos referidos a cada factor de riesgo de forma individual: education and aggression or violence; poverty and aggression or victim or violence; political rights and aggression or victim or violence; social rights and aggression or victim or violence; religiosity and aggression or victim or violence; conflict and aggression or victim or violence; masculine cultures and aggression or victim or violence, forced marriage and aggression or victim or violence; democracy and aggression or victim or violence; globalization and aggression or victim or violence. Age and aggression or victim or violence; income and aggression or victim or violence; employment and aggression or victim or violence; witness violence in childhood and aggression or victim or violence; number of children and aggression or victim or violence; forgiveness and aggression or victim or violence, bidirectionality and aggression or victim or violence, satisfaction or well being and aggression or victim or violence; attitudes and violence; emotions and violence; pregnancy and aggression or victim or violence y drug or alcohol abuse and aggression or victim or violence.

La búsqueda en la literatura identificó 198 estudios para su posible inclusión en esta revisión de los cuales 10 son meta-análisis (realizados a partir de estudios previos a los presentados en las tablas). Treinta y tres estudios fueron incluidos en las tablas entre los que encontramos, revisiones sistemáticas (3) y estudios primarios (30) que complementan la información hasta el momento recogida en las revisiones meta-analíticas. Fueron excluidos 155 artículos de esta revisión. Se aplicaron 4 criterios de exclusión (1) sólo se tomaron en cuenta los estudios que incluían factores de riesgo de violencia en la pareja, (2) se excluyen aquellas investigaciones que eran actas de congresos y las tesis doctorales (3) contenían muestras duplicadas, y (4) no contenían datos suficientes para obtener un tamaño del efecto.

Las variables de tratamiento se codificaron según (a) el factor de riesgo asociado al incremento de la VPI, (b) los niveles de agrupación propuestos desde la teoría socioecológica (nivel macroestructural, exo, micro y onto), y (c) el tipo de violencia sufrida: violencia física, psicológica, emocional, verbal o sexual (o de los distintos tipos combinados de violencia). En cuanto a las características de la muestra, se tuvo en cuenta: (a) el tamaño de la muestra, (b) el género (hombres y mujeres), y (c) tipo de población (parejas, estudiantes o población general).

\section{Resultados}

\section{Nivel de agrupación comunitario-societal}

En este nivel se analizaron los factores de riesgo de la violencia de género relacionados con el macro-sistema y el exosistema. Es decir, aquellos factores que tienen que ver con las creencias y los valores culturales que determinan la violencia de género, así como las estructuras formales e informales que influyen, conforman y determinan el ambiente en el que la persona se desenvuelve.

\section{Factores de riesgo asociados al macrosistema}

Los estudios más recientes diferencian entre factores de tipo comunitario e individual y analizan el impacto del contexto cultural en el incremento del riesgo a ser expuesto a violencia por parte de la pareja (Kaya y Cook, 2010). Entre los factores comunitarios encontrados destacan la falta de poder de las mujeres (Kishor y Johnson, 2004; Tokuç, Ekuklu y Avcioğlu, 2010), el contexto cultural y la globalización (véase Tabla 1 con los datos del meta-análisis realizado por Stith et al. en el año 2004 y de los estudios individuales).

$\mathrm{El}$ primer factor de riesgo de violencia sufrida por la mujer en la pareja, que está relacionado con la capacidad de empoderamiento, incluye el nivel educativo y el desarrollo económico del país (Kaya y Cook, 2010). La educación y el empleo se presentan como las dimensiones más importantes de empoderamiento de las mujeres, además de los derechos políticos y sociales igualitarios. Estos autores explican que, a medida que una sociedad se desarrolla económicamente y las mujeres adquieren los derechos políticos y sociales, y acceden al trabajo y la educación, existe una mayor posibilidad de escapar de la situación de violencia. Existe una relación entre la pobreza y la violencia familiar, así como entre el nivel educativo y socio-económico bajo y la violencia conyugal (Andersson, Cockcroft., Ansari, Omer, Chaudhry, Khan y Pearson, 2009; Echeburúa, Amor y De Corral, 2002; Tokuç et al., 2010). Otros estudios han encontrado que en los países en vías de desarrollo, los niveles de violencia contra mujeres embarazadas son mayores que en las sociedades más desarrolladas (Campbell, García-Moreno y Sharps, 2004; Kaya y Cook, 2010; Nasir y Hyder, 2003; Tokuç et al., 2010). Para esta asociación se han incluido explicaciones como son la relación entre el nivel de estrés y la pobreza (frustraciónagresión) (Dollard, Doob, Miller, Mowrer y Sears, 1939) o interpretaciones sobre las dinámicas de la lucha de clases (Martín-Baró, 1983). El empoderamiento incluye la participación de la mujer y su reconocimiento profesional. Según algunos estudios, el acceso de la mujer a un empleo reduce el riesgo de sufrir violencia (Villarreal, 2007). 
Tabla 1. Factores de riesgo asociados a la VPI a nivel socio-comunitario: Macrosistema.

\begin{tabular}{|c|c|c|}
\hline Estudio & Muestra & Evidencia empírica \\
\hline \multicolumn{3}{|l|}{ Empoderamiento } \\
\hline \multicolumn{3}{|l|}{ Nivel educativo: } \\
\hline \multicolumn{3}{|c|}{ Stith et al. $(2004)^{\mathrm{c}} k=13, N=4544, r=-.05, p<.001$} \\
\hline Kaya y Cook $(2010)^{\mathrm{b}}$ & $N=40$ paíse & sBajo nivel educativo asociado con mayores tasas de VPI, $r=-.69, p<.0001$. \\
\hline Tokuç et al. $(2010)^{\mathrm{a}}$ & $n=288 \mathrm{M}$ & $\begin{array}{l}\text { Más VPI en mujeres no escolarizadas, frente a las escolarizadas: } \chi^{2}=5.33, p<.05, O R=1.92,95 \% \\
\text { IC [1.08- 3.38] (Turquía). }\end{array}$ \\
\hline Anderson et al. (2009) ${ }^{\mathrm{a}}$ & $N=3430 \mathrm{M}$ & $\begin{array}{l}\text { Mayor probabilidad de VPI si la mujer no tenía educación formal que si tenía educación formal OR } \\
=1.42,95 \% \text { IC }[1.07-1.90] \text { (Pakistán). }\end{array}$ \\
\hline \multicolumn{3}{|c|}{ Pobreza-nivel desarrollo país } \\
\hline Tokuç et al. $(2010)^{a}$ & $n=288 \mathrm{M}$ & $\begin{array}{l}\text { Tasas de } 54 \% \text { de VPIF en PVD versus } 27 \% \text { en PD, } \chi^{2}=19.11, p<.001, \text { OR }=3.14,95 \% \text { IC [1.86- } \\
5.29 \text { ] (Turquía). }\end{array}$ \\
\hline
\end{tabular}

Kaya y Cook (2010) b $\quad N=40$ países El nivel de desarrollo del país, $r=-.08 p<.0001$, y la riqueza, $r=-.52, p<.0001$, están relaciona-

Derechos políticos y sociales

Sullivan et al. (2010) a $\quad N=103 \mathrm{M} \quad$ Las VPIF, VPIP, VPIS se relacionan con reacciones sociales de tipo negativo para la lucha contra la VPI $(r=.27, r=30, r=28, p<.01$, respectivamente) (Inglaterra).

Kaya y Cook $(2010)^{\mathrm{b}} \quad N=40$ países Los derechos políticos, $r=-.24, p<.0001$, y sociales, $r=-.45, p<.0001$ se asocian con más abandono de la situación de violencia.

Chan et al. (2011)a $\quad N=2225 \quad$ Recibir apoyo y seguridad por parte del estado reduce los niveles de VPI, OR $=2.52$, 95 \%, IC

Falta de autonomía de la mujer

Tokuç et al. (2010) $\quad \quad N=288 \mathrm{M} \quad$ Los valores patriarcales (decisiones que toma la familia $75 \%$ ), frente a otros valores que promocionan la libertad de decisión por parte de la mujer (ella y su familia 35\%, y ella sola 30.4\%), $\chi^{2}$ $=15.13, p<.0001, \mathrm{OR}=4.60,95 \%$, IC [1.42-14.80], $p<.05$, y la falta de autonomía (desempleo $(42.6 \%)$ vs. estar empleado (21.3\%), $\chi^{2}=12.53, p<.0001$ OR $=2,15,95 \%$ IC $\left.[1.06,4.37], p<.05\right]$ predicen VPI (Turquía).

Villarreal $(2007)^{a} \quad N=33709$ MEncuesta del Instituto Nacional de Estadística. Existe una asociación entre la falta de autonomía de la mujer y la VPI de $r=.20, p<.05$ (México).

Contexto cultural

Creencias religiosas

Kaya y Cook (2010) b

Guerras- conflictos

Clark et al. (2010)a

$n=40$ países Nivel de fragmentación religiosa asociado con la VPI, $r=.15, p<.0001$.

$N=3510 \mathrm{M}$ Las mujeres cuyos maridos fueron expuestos directamente a violencia colectiva reportaron más probabilidades de VPIF OR $=1.89,95 \%$ IC $[1.29-2.76], p<.01$ y de VPIS, OR $=2.23,95 \%$ IC

probabilidades de VPIF OR $=1.89,95 \%$ IC $[1.29-2.76], p<.01$
$[1.49-3.35], p<.05$ frente a los que no fueron expuestos (Gaza).

Saile et al. (2013)

$N=235$ pa-Los países de escasos recursos y afectados por la guerra tienen alto riesgo de VPI. VPIP: $F(17,217)=$ rejas $2,61, p<.01$; VPIF: $F_{(17,217)}=4.76, p<.01$; Aislamiento total: $F_{(17,217)}=4.90, p<.001$ (Uganda).

Stewart y Brown (2010) b $N=6$ países Existe una relación entre la violencia política y aumento de las tasas de VPI $(r=.78, p<.05)$.

Culturas favorables a la violencia

Archer $(2006)^{\mathrm{a}} \quad N=52$ países Actitudes sexistas $(\mathrm{HS}, r=.53, p<.05)$ y estar de acuerdo con golpear a la esposa $(r=.58, p<.05)$

se asociaron a VPI.

Pérez et al. $(2002)^{\mathrm{a}} \quad N=316 \mathrm{H}$ yUxoricidio asociado al bajo desarrollo social, $r=.35, p<.05$.

$\mathrm{M}$

Moya et al. (2002) $\quad \quad N=15000 \mathrm{~A}$ mayor IDH menor SH, $r=-.77, \mathrm{p}<.05$ y SB, $r=-.86, p<.05$. SH mayor en sociedades con ba(19 países) jo IDH, $r=-.66, p<.02$, y con más distancia jerárquica, $r=.50, p<.01$.

Matrimonio impuesto

Tokuç et al. (2010)a

$N=288 \mathrm{M}$ Menos probabilidad de VPI, cuando la mujer hace una elección libre del matrimonio (30.4\%) que cuando es forzada (75\%, la toma la familia): $O R=4.60, p=.011,95 \%$, IC [1.42-14.80]. No hay diferencias cuando la elección es conjunta $(35.2 \%$, por la familia y propia) $O R=1.52,95 \%$, IC [.82$2.80], p=.18$ n.s.

Democratización del Estado

Weldom (2002) a $\quad N=36$ países Las políticas y el papel activo de las agencias disminuyen las tasas de VPI, $r=.42, p<.001$.

Kaya y Cook $(2010)^{a} \quad N=40$ países La democratización está asociada con menores tasas VPI, $r=-.36, p<.0001$.

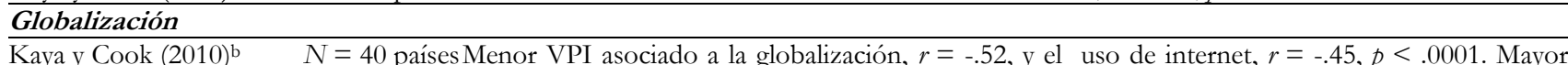
VPI si se depende de PD, $r=.33, p<.0001$.

$\bar{N}=$ sujetos de los estudios; $n=$ tamaño de la submuestra; $K=$ número de estudios; $r=$ tamaño del efecto medio; OR= Odds ratio; IC $=$ intervalo de confianza; M: mujer; H: hombre; VPI: Violencia por parte de la Pareja Íntima; VPIF: Violencia Física por parte de la Pareja Íntima; VPIS: Violencia Sexual por parte de la Pareja Íntima; VPIP: Violencia Psicológica por parte de la Pareja Íntima; PVD: países en vías de desarrollo; PD: países desarrollados; IDH: Índice de desarrollo humano; BS: Sexismo Benévolo, HS: Sexismo Hostil (Glick y Fiske, 1996).

${ }^{a}$ estudios primarios; ${ }^{b}$ revisiones sistemáticas, ${ }^{\mathrm{c}}$ meta- análisis. 
En segundo lugar, el contexto cultural también puede influir sobre el nivel de empoderamiento de las mujeres. Por ejemplo, en países como Kenia (Lawoko, Dalal, Jiayou y Jansson, 2007) y en Pakistán (Anderson et al., 2009) el nivel de instrucción y el acceso al trabajo de las mujeres, en vez de protegerlas, predicen un incremento del riesgo de sufrir violencia. Uno de los elementos del contexto cultural son las creencias religiosas (Wendt, 2008). Kaya y Cook (2010) demostraron que no es la adscripción a una religión u otra la que predice un mayor riesgo de violencia, sino que es la intensidad o nivel de adherencia a estas creencias las que favorecen la violencia contra las mujeres.

También los estados de guerra, los conflictos étnicos y religiosos y la militarización de la sociedad incrementan el riesgo de violencia en la pareja: las mujeres que viven en países de escasos recursos afectados por guerras recientes tienen más riesgo de sufrir violencia por parte de la pareja (Adelman, 2003). En zonas como Uganda (Saile, Neuner, Ertl y Catani, 2013), Paquistán (Ali, Asad, Mogren y Krantz, 2011; Clark, Everson-Rose, Suglia, Btoush, Alonso y HajYahia, 2010) y Etiopía (García-Moreno, Jansen, Ellsberg, Heise y Watts, 2006), altamente afectadas por guerras pasadas, un año después del conflicto se incrementaron las tasas de abuso psicológico, físico y sexual contra las mujeres (Stewart y Brown, 2010).

También existen culturas masculinas que legitiman la violencia cultural y justifican la agresión física interpersonal. Estas sociedades desarrollan la "cultura del honor" tradicionalmente asociada con mayores niveles de violencia general y hacia la pareja (Ubillos, Martin-Beristain, Garaigordobil y Halperin, 2008). Además, la masculinidad cultural está relacionada con mayores tasas de violencia hacia la pareja (Archer, 2006; Páez y Ubillos, 2004). Otros estudios encontraron una asociación entre los casos de uxoricidio y las culturas jerárquicas más tradicionales, el bajo desarrollo social y la cultura colectivista (oposición entre la cultura del honor y la liberación de la mujer) (Moya, Páez, Glick, Fernández y Poeschl, 2002; Pérez, Páez, Navarro y Arias, 2001).

No menos importante en el contexto cultural es lo que se refiere a la libre elección de la pareja, el matrimonio y el estatus marital. En algunas sociedades de Asia, Oriente Medio y África, las condiciones para la elección del matrimonio y la pareja son impuestas por las familias y los hombres, y están asociadas a mayores tasas de violencia contra las mujeres, como es el caso de Pakistán (Anderson et al., 2009) y Turquía (Tokuç et al., 2010). En zonas como Bangladesh la demanda de la dote en el matrimonio aumentó la probabilidad de violencia hacia la mujer en las zonas rurales (Naved, Azim, Bhuiya y Persson, 2006). Además, en las sociedades donde se practicaba la poligamia había mayores tasas de violencia contra la mujer (Anderson et al., 2009; Arzu y Pinky, 2001).

Otro aspecto a destacar del contexto cultural es la democratización del Estado. La existencia de un sistema democrático y la protección de los derechos humanos ayudan a prevenir la violencia de género. Los países que han sido demo- cratizados generan respuestas oficiales a la violencia contra las mujeres y las políticas o agencias desempeñan un papel fundamental (Weldon, 2002).

Por último, la globalización o integración de un país en la economía mundial en parte han facilitado el descenso de las tasas de violencia (Kaya y Cook, 2010). Sin embargo, la globalización no siempre disminuye estas tasas, ya que también puede obstaculizar la autonomía y coartar la libertad de las mujeres. La globalización ha creado nuevas oportunidades para ejercer violencia, como son el tráfico de mujeres y el turismo sexual (Radford y Tsutsumi, 2004). Además, la inclusión de un país dentro de la economía mundial no reduce los niveles de violencia física en la pareja y no favorece la independencia de la mujer si se depende de países más desarrollados (Kaya y Cook, 2010).

\section{Factores asociados al exosistema}

Entre los elementos de carácter comunitario, se distinguieron 3 factores: ser menor de edad, tener ingresos bajos y estar desempleado (Stith et al., 2004) (véase Tabla 2).

Algunos estudios encuentran que tener menor edad (menos de 34 años) está asociado con el riesgo de ser víctima de violencia por parte de la pareja (Chan, Tiwari, Fong, Leung, Brownridge y Ho, 2011; Hedin, 2000). La mayor parte de las investigaciones constatan que la prevalencia de la violencia en la pareja disminuye a medida que la edad aumenta (Naved et al., 2006; Timmons y O'Leary, 2004). Además se encuentra que las agresiones físicas dentro de la pareja seguirían un patrón en U invertida (O’Leary, 1999), de modo que la implicación en comportamientos violentos era muy baja en las parejas más jóvenes y en las más adultas, encontrándose los niveles más altos entre los 22 y los 25 años (Archer, 2000; Capaldi, Shortt y Kim, 2005; Riggs et al., 2000; Timmons y O'Leary, 2004). En contraste, otros estudios muestran que las mayores tasas de violencia se producen en el primer grupo de edad (de 18 a 29 años) disminuyendo posteriormente de forma progresiva en el segundo (de 30 a 50 años) y aumentado de nuevo en el tercer grupo de edad (>50 años) (Graña, Rodríguez y Peña, 2009; Nocentini, Menesini y Pastorelli, 2010).

El riesgo de ser víctima de violencia también está relacionado con los bajos recursos y la dependencia económica (Expósito, Herrera, Moya y Glick, 2010; Stith et al., 2004). La violencia impide a las mujeres buscar empleo y sin independencia económica, no tienen recursos para abandonar la relación abusiva (Tokuç et al., 2010; Chan et al., 2011).

Por último se añade como factor de riesgo ser testigo de violencia o haber experimentado abusos en la infancia (Chang et al., 2011) Sin embargo no todos los estudios confirman que vivir en un hogar violento es predictor de ser agresor en la edad adulta (Kim, Talbot y Cicchetti, 2009; Carboné -López y Kruttschnitt 2010). 


\section{Nivel de agrupación contextual}

En este segundo apartado se incluyen los factores de riesgo de la violencia de género relacionados con el microsistema (o contexto en el que se produce el abuso) y el ontoge- nético. Por tanto, aquellos factores que tienen que ver con el patrón de interacción y la estructura del sistema familiar, así como con la historia de desarrollo y aprendizaje del individuo.

Tabla 2. Factores de riesgo asociados a la VPI a nivel socio-comunitario: Exosistema

\begin{tabular}{|c|c|c|}
\hline Estudio & Muestra & Evidencia empírica \\
\hline \multicolumn{3}{|c|}{ Edad: Stith et al. (2004) $k=17, N=5832, r=-.07, p<.001$, } \\
\hline Chan et al. (2011)a & $N=2225 \mathrm{H}$ у M & $\begin{array}{l}\text { Mayores niveles de VPI con menos de } 34 \text { años, } O R=2.113, \mathrm{p}<.05,95 \% \text { IC }[1.141 \text { - } \\
3.195 \text { ] (China). }\end{array}$ \\
\hline \multicolumn{2}{|c|}{ Timmons y O’Leary (2004)a $N=203 \mathrm{M}$} & $\begin{array}{l}\text { Existe una relación entre tener menor edad y la probabilidad de que suceda algún acto de } \\
\text { VPIF, } r=-.82, p<.001 \text {. (USA) }\end{array}$ \\
\hline O’Learly (1999)a & $N=203 \mathrm{M}$ & La correlación VPIF y edad fue $r=-.82, p<.001$ (USA). \\
\hline Nocentini et al. (2010)a & $N=86$ adolescentes & $\begin{array}{l}\text { Existe una relación entre los niveles de victimización (violencia física) y tener más edad: } \\
16 \text { años }(r=.64, p<.001) \text {, con } 17(r=.69, p<.001) \text { y con } 18(r=.86, p<.001) \text {. }\end{array}$ \\
\hline \multicolumn{3}{|c|}{ Recursos económicos. Stith et al. (2004)c Ingresos: $k=10, N=4097, r=-.04, p<.001$. Empleo: $k=6, N=2979, r=.05$, n.s. } \\
\hline Tokuç et al. (2010)a & $N=288 \mathrm{M}$ & $\begin{array}{l}\text { Existe un vínculo entre la violencia y la dependencia económica }(n=83,42.6 \%) \text { frente a } \\
\text { las mujeres que son independientes económicamente }(n=20,21.3 \%), O R=2.15,95 \% \\
\text { IC }[1,06-4,37], \chi^{2}=12.53, p<.0001 \text {. }\end{array}$ \\
\hline Chan et al. (2011)a & $N=2225 \mathrm{M}$ & El desempleo aumenta la probabilidad de VPI, OR = .92, IC 95\% [.41- 2.03] n.s. \\
\hline \multicolumn{3}{|c|}{ Ser testigo de violencia en la infancia } \\
\hline Kim et al., (2009). ${ }^{\mathrm{a}}$ & $\begin{array}{l}N=129 \mathrm{M} \text { y sus hi- } \\
\text { jos }\end{array}$ & Ser testigo de VPI en la \\
\hline Chan et al. (2011)a & $N=2225 \mathrm{H}$ y M & $\begin{array}{l}\text { Ser testigo de violencia en la infancia aumenta el riesgo de la VPI en la edad adulta. OR } \\
=2.849,95 \% \text { IC }[1.482-5.479] p<.01\end{array}$ \\
\hline
\end{tabular}

$N=$ sujetos de los estudios; $K=$ número de estudios; $r=$ tamaño del efecto medio; $O R=$ Odds ratio; IC $=$ intervalo de confianza; $\mathrm{R}^{2}=$ coeficiente de determinación; $\beta=$ peso de la variable; n.s.: no significativo; M: mujer; H: hombre; VPI: Violencia por parte de la Pareja Íntima VPIF: Violencia Física por parte de la Pareja Intima; VPIS: Violencia Sexual por parte de la Pareja Intima; VPIP: Violencia Psicológica por parte de la Pareja Intima.

${ }^{\mathrm{a}}$ estudios primarios; ${ }^{\mathrm{b}}$ revisiones sistemáticas, ${ }^{\mathrm{c}}$ meta- análisis.

\section{Factores asociados al contexto de abuso o microsistema}

Entre los factores de riesgo en el nivel del microsistema se incluyen los que ocurren en el contexto de abuso, como son: el número de hijos, el estatus marital, la satisfacción con la pareja y la ejecución de actos violentos (Stith et al., 2004) (véase Tabla 3).

Numerosos estudios han comprobado la relación que existe entre mayor número de hijos y el riesgo de violencia contra la mujer (Hazen y Soriano, 2007; Kaya y Cook, 2010; Ruiz-Pérez, Mata-Pariente y Plazaola-Castaño, 2006). Tener grandes cargas familiares incrementa el riesgo de las mujeres de sufrir violencia por parte del compañero (Tokuç et al., 2010; Vives-Cases, Álvarez-Dardet, Gil-González, Torrubiano-Domínguez, Rohlfs y Escribà-Agüir, 2009).

Estar casada es un factor de riesgo que predice mayores niveles de victimización en las mujeres (Chan et al., 2011; Vives-Cases et al., 2009). Algunas investigaciones señalan que las mujeres más expuestas a las agresiones masculinas no son las casadas, sino las separadas y las solteras (Vives-Cases et al., 2009). A este respecto, Cáceres (2004) encontró que se producía un incremento de la violencia global una vez iniciado el periodo de separación. Otros autores hallaron que la duración de la relación es la que aumenta la probabilidad de violencia en la pareja. Las tasas de violencia física y psicológica eran mucho más bajas en las parejas que no estaban casadas y, cuanto mayor era el tiempo de relación, mayores eran los niveles de conflictividad (Carboné-López y
Kruttschnitt, 2010). En contra, otros estudios han encontrado que la duración de la relación y el estado civil no tienen ningún efecto sobre la violencia (Chen y Whitte, 2004).

Además, con el tiempo en las parejas se produce un deterioro del grado de armonía general. La función del matrimonio en relación a la violencia en la pareja es altamente mediada por los niveles de satisfacción marital (Cáceres, 2004; Cáceres y Cáceres, 2006). Así, a mayor satisfacción en la relación de pareja, menor probabilidad de sufrir violencia de género (Stith, Green, Smith y Ward, 2008).

Otro factor importante en la satisfacción se refiere a la congruencia de creencias culturales: las parejas jóvenes que tienen una percepción similar respecto a la cultura del honor (ya sea alta o baja la importancia otorgada a este aspecto) se sienten más satisfechas en su relación de pareja (LopezZafra y Rodríguez, 2008). El apoyo de la pareja funciona de manera similar en todas las culturas y está relacionado con la satisfacción marital, esto es, a mayor apoyo mutuo percibido mayor satisfacción (Cramer, 2004). Estos argumentos apoyan las tesis feministas, en cuanto demuestran que la capacidad de llegar a acuerdos se asocia con menores niveles de violencia en la pareja (Cáceres, 2004).

La tendencia a hacer interpretaciones relativamente optimistas de experiencias indeseables se ha asociado con mayores niveles de satisfacción marital. (McNulty y Fincham, 2012). Sin embargo estos efectos estaban mediados por la gravedad de la agresión. Realizar atribuciones más externas, menos globales y menos estables sobre conductas negativas 
y leves de las parejas mantiene la satisfacción marital cuatro años después (Karney y Bradbury, 2000). Cuando eran graves, realizar una atribución benévola sobre la conducta negativa se asociaba con menor satisfacción y es un factor potencial de violencia que mantiene la situación de violencia, siendo este estilo de atribución desadaptativo (McNulty, O'Mara y Karney, 2008).

De igual modo, la satisfacción marital se asocia con el perdón por parte de la esposa de conductas hostiles, porque mejora la percepción subjetiva en la comunicación con el marido 12 meses más tarde (Fincham, Beach y Dávila,
2007). Sin embargo, el nivel de tolerancia se ha asociado con más probabilidades de volver con la pareja violenta (Gordon, Burton y Porter, 2004). La tendencia de las mujeres a tener comportamientos crueles cuando hay problemas graves (por ejemplo, rechazo, crítica) predice mayor satisfacción para ambos miembros de la pareja, en comparación con los problemas menos graves (McNulty y Russell, 2010). Sin embargo, la bondad (por ejemplo, entendida como conducta de apoyo) también ha sido asociada con mayores niveles de satisfacción marital siempre que responda a una necesidad de la pareja (Lucas, Parkhill, Wendorf, Imamoglu et al., 2008).

Tabla 3. Factores de riesgo asociados a la VPI a nivel contextual: Microsistema.

Estudio Muestra $\quad$ Evidencia empírica

Número de hijos-cargas familiares. Stith et al. (2004) ${ }^{\mathrm{c}}$ número hijos, $k=22, N=4774, r=.06, p<.001$

Hazen y Soriano $(2007)^{\mathrm{a}} \quad N=292 \mathrm{M}$

VPIS asociado con mayor número de hijos. 1 o 2 hijos $(n=175,59.9 \%)$ OR $=.28,95 \%$ IC $[.10, .76] p<.05$, en comparación con las que no tienen hijos ( $n=46,15.8 \%$ ) (USA)

Kaya y Cook $(2010)^{\mathrm{b}}$

Tokuç et al. (2010)a

$N=40$ países Tasas de fertilidad asociadas con un incremento del VPI, $r_{(40)}=.78, p<.001$ ) (USA)

Vives-Cases et al. (2009)a

$N=102 \mathrm{M}$

Mujeres con hijos $(n=90,38.6 \%)$ más VPI, vs sin hijos $(n=12,22.2 \%), \chi^{2}=4.62, \mathrm{OR}$ $=.29,95 \%$, IC [.16-.53], $p=.032$ (Turquía).

$N=13000 \quad$ Tasas más altas de VPI si se tienen tres o más menores a su cargo $(\mathrm{n}=12,9.4 \%)$, que si $(n=128 \mathrm{M}) \quad$ no tienen hijos $(n=66,51.6 \%), \mathrm{OR}=3.5,95 \%$ IC [1.78-6.90], $p<.0001$ (España).

\section{Estado civil}

Vives-Cases et al. (2009)a

Cáceres (2004)a $\quad N=166(76 \mathrm{H} \mathrm{y} \mathrm{Las} \mathrm{diferencias} \mathrm{entre} \mathrm{novios} \mathrm{y} \mathrm{matrimonios} \mathrm{son} \mathrm{estadísticamente} \mathrm{significativas} \mathrm{en} \mathrm{VPI}$

$N=13000 \mathrm{H}$ y M Mayores tasas VPI si está separada o divorciada $(n=20,15.6 \%), \mathrm{OR}=2.81,95 \% \mathrm{IC}$ $(n=128 \mathrm{M}) \quad[1.89-4.97]$ que si estaba soltera $(n=34,26.6 \%)$ (España). $90 \mathrm{M}) \quad[F(1,58)=32,63 ; p<.0001], \operatorname{VPIF}[F(1,58)=31,65 ; p<.0001] \operatorname{VPIP}[F(1,58)=$ 42,$64 ; p<.0001$ (España).

Duración de la relación

Carboné y Kruttschnitt, $(2010)^{a} N=162 \mathrm{M} \quad$ La duración de la relación es la que aumenta la probabilidad de VPI. $\mathrm{R} 2=1.17 \mathrm{p}<.0001$.

Satisfacción marital

Cáceres (2004)a

$N=166(76 \mathrm{H}$ y La correlación entre el grado de violencia total y el grado de armonía relacional es alta y $90 \mathrm{M}) \quad$ negativa, $r=-.56, p<.01$. Menos niveles de VPI, si se llega a acuerdos $\left(M_{\text {bajos niveles violen- }}\right.$ cia $\left.=15, D T=6, M_{\text {altos niveles violencia }}=28, D T=13, F_{(166)}=17.1, p<.001\right)$ (España).

Cáceres y Cáceres $(2006)^{a} \quad N=60 \mathrm{M} \quad$ La correlación entre el índice de violencia y la armonía relacional es de $r=-.72, p<.01$

(España)

McCollum y Stith (2008) $\quad N=8628 \quad$ La menor satisfacción marital predice VPI, $r=-0,27, p<.05$ (USA).

Cramer (2004)a $\quad \begin{aligned} & K=32 \\ & N=80 \mathrm{M} \mathrm{y} 27 \mathrm{H} \mathrm{La} \text { baja satisfacción marital correlaciona de manera positiva con los conflictos en la pare- }\end{aligned}$ ja, $r=.32, p<.001$, y el grado de apoyo familiar, $r=.44, p<.001$.

McNulty et al. (2008) a $\quad N=251$ nuevos Atribuciones benévolas sobre la conducta negativa se asociaba con mayor satisfacción, $r$

\section{Perdón}

Fincham et al. (2007)a $\quad N=96$ parejas $\quad$ La satisfacción marital, $r_{(96)}=.39, p<.01$, y el perdón de la esposa, $r(96)=.20, p<.05$, influyen en la percepción subjetiva de mejoras en la comunicación con el marido 12 meses más tarde (USA).

Gordon et al. (2004)a $\quad N=121 \mathrm{M}$ casa Hacer atribuciones menos maliciosas (más tolerantes) sobre la VPI está asociado con McNulty y Russell (2010)a acogida más probabilidades de volver con la pareja violenta, $r_{(121)}=-.43, p<.001$ (USA). $N=72$ parejas deLas mujeres con comportamientos crueles cuando hay problemas graves (por ejemplo, recién casados. rechazo, crítica) predicen mayor satisfacción para ambos miembros de la pareja, $r=.57, p$ $N=172 \mathrm{M}<.001$, en comparación con los problemas menos graves, $r=.08$, tendencial (USA).

Bidireccionalidad Stith et al (2004) $)^{\mathrm{c}}$ violencia hacia la pareja $k=5, N=652, r=.41, p<.001$

Cáceres $(2004)^{\mathrm{a}} \quad N=166(n=$ Tasas similares de violencia entre $\mathrm{H}(M=35,71, D T=24,09)$ y $\mathrm{M}(M=45,73, D T=$ $76 \mathrm{H}$ y $90 \mathrm{M}) \quad 23,46), p<.96$ n.s.

Moral et al. (2011) a $\quad N=233$ M y 177 Existe una correlación positiva y significativa entre la violencia ejercida y recibida $(r=$ $\mathrm{H} \quad .59, p<.01)$. (Monterey)

$N=$ sujetos de los estudios; $r=$ tamaño del efecto medio; $K=$ número de estudios; $O R=$ Odds ratio; IC= intervalo de confianza; M: mujer; H: hombre; VPI: Violencia por parte de la Pareja Íntima; VPIF: Violencia Física por parte de la Pareja Íntima; VPIS: Violencia Sexual por parte de la Pareja Íntima; VPIP: Violencia Psicológica por parte de la Pareja Íntima.

${ }^{\mathrm{a}}$ estudios primarios; ${ }^{\mathrm{b}}$ revisiones sistemáticas, ${ }^{\mathrm{c}}$ meta- análisis. 
Por último, en la mayor parte de los estudios donde participan ambos sexos no existen diferencias significativas en los promedios de victimización y agresión (Moral, Rosales, Loving y Martínez, 2011). De hecho, la mayoría de los estudios sobre violencia en las relaciones de pareja han encontrado que el uso de la violencia tiende a ser recíproco. Esta explicación excluye la violencia que las mujeres utilizan como método de defensa ante una agresión por parte de su pareja (Cáceres, 2004; Stith et al., 2004) y se incluye dentro de las dinámicas familiares negativas producto del estrés (Dutton, 2006).

\section{Factores asociados al nivel ontogenético}

Destaca como factores individuales asociados a ser víctimas de violencia: las actitudes a favor de la violencia, el afecto negativo (culpa, vergüenza, miedo y la depresión), las conductas hostiles, el embarazo y el abuso de alcohol y drogas (Stith et al., 2004) (véase Tabla 4).

Hay actitudes a favor de la violencia que funcionan como elementos mediadores entre las situaciones de conflicto y su forma de gestionarlos (Nayak, Byrne, Martin y Abraham, 2003). La justificación de la violencia, la adaptación, la tolerancia y la falta de empatía del agresor sobre la víctima se asocian a un mayor riesgo de sufrir violencia (Moral et al., 2011).

$\mathrm{El}$ afecto negativo puede servir como motivador del conflicto y la agresión en la pareja (Niedenthal, Krauth-Gruber y
Ric, 2006; Valor-Segura, Expósito y Moya, 2010). A mayor culpa y vergüenza más riesgo de violencia. La vergüenza genera malestar, confusión en la víctima e impide la acción, y la culpa cumple una función reparadora sobre las conductas negativas del agresor y mantiene a la mujer en la situación de maltrato (Etxebarria, Ortiz, Conejero y Pascual, 2009; Sheikh y Janoff-Bulman, 2010). Por otra parte, el miedo se asocia a la huida y aleja a las mujeres de la situación de violencia, sin embargo, en situaciones de violencia crónica hace que la mujer se mantenga en la relación y favorece el ejercicio de nuevas conductas violentas (Echeburúa et al., 2002; Folkman, 2010; Moral et al., 2011; Stith et al., 2004). La depresión es parte de la respuesta de la víctima. Supone más uso del cariño y búsqueda de ayuda, frente a la ira que implica la superación de los obstáculos y un afrontamiento de tipo instrumental (Páez, Martínez, Sevillano, Mendiburu y Campos, 2012).

Por otro lado, se considera que el embarazo es un factor de riesgo. Los niveles de agresión física y sexual son mayores en el grupo de mujeres embarazadas - la sufre el $8 \%$ de mujeres no embarazadas frente a un $18 \%$ para aquellas que sí lo estaban (Campbell et al., 2004; Chan et al., 2011).

Por último, respecto al abuso de sustancias, el consumo de alcohol y otras drogas actúan como factores de riesgo en la violencia de género - en ambos miembros de la pareja (Hazen y Soriano, 2007; Kliewer y Zaharakis, 2013).

Tabla 4. Factores de riesgo asociados a la VPI a nivel contextual: Ontogenético.

Estudio Muestra Evidencia empírica

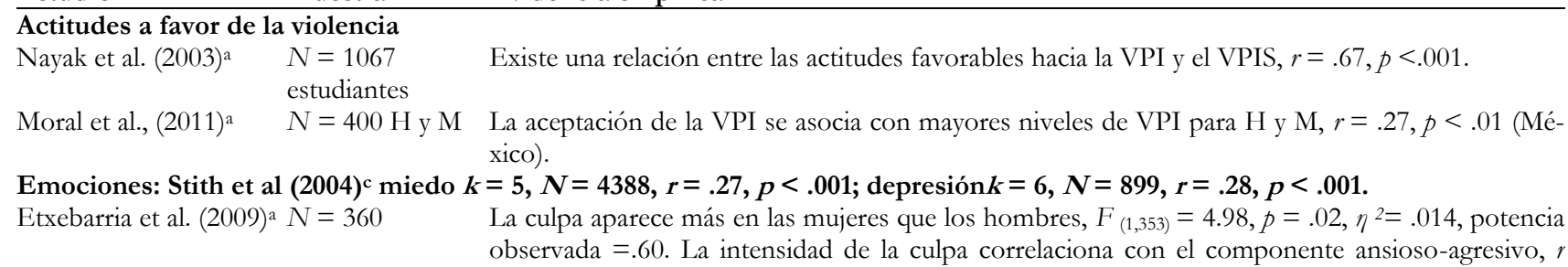
${ }_{(272)}=.46, p<.001,95 \%, \mathrm{IC}= \pm .40$. (España)

Valor-Segura et al. $\quad N=142(75 \mathrm{M}$ yEl miedo está asociado a situaciones de intimidación física por parte de la pareja, $F$ (1,136) $=33.06$, $(2010)^{\mathrm{a}}$

Moral et al. (2011) a $\quad N=233 \mathrm{M} \mathrm{y} 177 \mathrm{El}$ miedo se asocia con el uso de un estilo de afrontamiento pasivo, $r(400)=.32, p<.01(\mathrm{Monte}-$ $\mathrm{H}$ rey).

Embarazo

Chan et al. (2011)a $\quad N=2225 \quad$ La VPI, está asociado con estar embarazada, OR $=2.60,95 \%$ IC [1.36-4.97], en comparación con las no embarazadas (China).

Kaya y Cook $(2010)^{\mathrm{b}} \quad N=40$ países $\quad$ El embarazo estás asociado con la VPI, $r=.78, p<.0001$.

Stith et al (2004): consumo alcohol, $k=11, N=7084, r=.13, p<.001$

Hazen y Soriano (2007) ${ }^{\mathrm{a}} \mathrm{N}=29 \mathrm{M}$ latinas Abuso de sustancias $(n=54,19.4 \%)$ asociado a más VPIF, OR $=2.59,95 \%$ IC $[0.58-3.40], p<$ .05; (USA).

Kliewer y Zaharakis, $\quad N=318 \mathrm{M} \quad$ El abuso de alcohol está asociado con un incremento en el riesgo de VPI, $r=.15, p<.05, \mathrm{y}$ con $(2013)^{\mathrm{a}}$ el de drogas, $r=.16, p<.05$.

$N=$ sujetos de los estudios; $r=$ tamaño del efecto medio; $K=$ número de estudios. $O R=$ Odds ratio; IC= intervalo de confianza; M: mujer; H: hombre; VPI: Violencia por parte de la Pareja Íntima; VPIF: Violencia Física por parte de la Pareja Íntima; VPIS: Violencia sexual por parte de la Pareja Íntima; VPIP: Violencia Psicológica por parte de la Pareja Íntima.

${ }^{\mathrm{a}}$ estudios primarios; ${ }^{\mathrm{b}}$ revisiones sistemáticas, ${ }^{\mathrm{c}}$ meta- análisis. 


\section{Discusión}

Esta revisión bibliográfica muestra de manera ordenada en base a una estructura lógica fundamentada en el modelo socio-ecológico los factores que se asocian con el riesgo de sufrir violencia de género según las investigaciones más recientes. En el nivel macro-social destacan como factores de riesgo relacionados con el empoderamiento y el contexto cultural: la falta de educación $(r=-69, p<.0001)$, el bajo desarrollo económico del país $(r=-.52, p<.0001)$, derechos sociales y políticos bajos $(r=-.45, p<.0001)$, la falta de autonomía de las mujeres y la baja democratización del estado $(r=$ $.36, p<.0001)$. Estos resultados sugieren que a medida que una sociedad se desarrolla económicamente y las mujeres adquieren los derechos políticos y sociales y acceden al trabajo y la educación, incrementado su autonomía, existe una mayor posibilidad de escapar de la situación de violencia (Villareal, 2007).

Además, la asociación entre fundamentalismo religioso y violencia es coherente con la idea que las religiones patriarcales crean un entorno social donde las mujeres son culturalmente dependientes de los hombres y donde la violencia contra las mujeres es legitimada y aceptada por la sociedad (West, 1999). La asociación entre violencia colectiva (guerras en el pasado y presente), las culturas masculinas y del honor y la violencia de pareja indica que existe una cultura de la violencia que resulta normativa e instrumental.

Estos resultados son coherentes con la correlación entre las actitudes positivas hacia el uso de la violencia, las actitudes tradicionales sobre los roles de género de las mujeres y la perpetración de actos violentos $(r=.53$ y $r=.58$ respectivamente, $p<.0001)$. En culturas masculinas tradicionales y del honor tiene lugar un proceso de idealización de la violencia que la define como una forma deseable de conducta, como una manera de manifestar la propia identidad, defender la autoestima personal y mantener la superioridad del hombre dentro de la familia (Abramsky, Watts, García-Moreno, Devries, Kiss, Ellsberg, Ellsberg, Jasen y Heise, 2011; Jewkes, 2002). Además, aunque con menor evidencia empírica, la exposición a la violencia relacionada con la guerra (experiencia de violencia organizada) hace a las personas más vulnerables para la comisión o perpetración de actos violentos contra la pareja y la re-victimización explica el aumento de las tasas de violencia familiar tras un conflicto (Catani, Schauer y Neuner, 2008).

Finalmente la asociación entre integración de un país en la economía mundial y menor violencia sugiere que la globalización ha facilitado la promoción de las mujeres, la apertura a mayores oportunidades de empleo, el acceso a la información y el exterior y la mayor expansión en materia de sensibilización, aunque también ha abierto nuevas posibilidades para la explotación sexual (Kaya y Cook, 2010).

Entre los factores de riesgo comunitarios la menor edad $\left(r_{(5832)}=-.05, p<.001\right)$, tener ingresos bajos $\left(r_{(4544)}=.04\right.$, $p<.001)$-aunque no estar desempleado- y haber sido testigo $\mathrm{y}$ víctima de agresión en la infancia $\left(r_{(1567)}=.24, p<.0001\right)$, se asociaron débilmente a ser víctima de violencia. El vínculo entre la violencia y la dependencia económica es circular (Stith et al., 2004; Tokuç et al., 2010). Por un lado, la amenaza y el miedo a la violencia impiden a las mujeres buscar empleo, aceptar puestos de trabajo con salarios superiores a los de su pareja o las obliga a dedicarse exclusivamente a las tareas del hogar (Expósito et al., 2010). Y a su vez, sin independencia económica, las mujeres no tienen ningún poder para escapar de una relación abusiva (Schuler, Hashemi, Riley y Akhter, 1996). En este nivel, tres meta-análisis encuentran una relación entre la violencia de género y presenciar o experimentar violencia familiar en la infancia (Riggs et al., 2000; Stith et al., 2000; Sugarman y Hotaling, 1997) aunque hay que destacar que alrededor de dos tercios de los maltratados o expuestos a la violencia no se transforman en agresores (Kim et al., 2009).

Entre los factores de riesgo del microsistema hay una asociación débil entre el número de hijos, las cargas familiares (cuidar abuelos, vivir con otros familiares) y violencia $\left(r_{(4774)}=.06, p<.001\right)$, lo que sugiere que la presencia de hijos y otros familiares son un elemento de estrés añadido en la relación que funciona como factor de riesgo (Martín, 1999).

El efecto de estar casado (frente a ser soltero) y la mayor victimización se explican por el carácter de idealización y la falta de tiempo en parejas recién formadas para haber percibido grandes discrepancias (Cáceres y Cáceres, 2006). La relación entre mayor duración de la pareja y violencia se explica porque con el tiempo en las parejas se produce un deterioro del grado de armonía general, de la satisfacción con la relación, de la cohesión y de la capacidad para expresar afecto y llegar a acuerdos.

Congruentemente con esto, la violencia en la pareja se asocia a bajos niveles de satisfacción marital (Cáceres, 2004; Cáceres y Cáceres, 2006). Interpretaciones más benevolentes de conductas negativas leves se asociaban al bienestar, porque evitan la generalización de una imagen negativa de la pareja e impiden la escalada de conflicto. Paradójicamente, estas interpretaciones benignas de agresiones graves son un factor de malestar, porque minimizan lo ocurrido y mantienen la exposición a la situación negativa. Igualmente, perdonar conductas hostiles se asocia a la satisfacción, aunque también a volver con la pareja violenta. Interacciones bondadosas aunque también crueles se han asociado a la satisfacción - sin embargo la evidencia es limitada. Finalmente la violencia tiende a ser recíproca y en contra de los estereotipos el nivel de agresión es similar en ambos sexos y es el factor de riesgo con más peso (Stith et al., 2004).

Entre los factores de carácter ontogenético o individual encontramos la depresión $(r(899)=.28, p<.001)$, el miedo $\left.r_{(4388)}=.27, p<.001\right)$, el embarazo y el consumo de alcohol $\left(r_{(7084)}=.13, p<.001\right)$. Hay menos evidencia de los efectos de la culpa y la vergüenza.

El miedo como respuesta a la violencia puede funcionar de forma ambivalente. Por un lado, puede ser una forma de afrontamiento activo si se asocia a la huida y evitación y aleja 
a las mujeres de la situación de violencia. Por otro lado, si el miedo aparece en situaciones de violencia continuada puede suponer un aprendizaje y habituación a la situación de amenaza de violencia (Echeburúa et al., 2002). Esta respuesta refuerza en el agresor el ejercicio de nuevas conductas violentas (Folkman, 2011; Moral et al., 2011; Stith et al., 2004). Esto se debe a que esta emoción debilita a la víctima, destruye su autoestima y la hace más vulnerable ante nuevas agresiones (Femat, 2008; Niedenthal et al., 2006; Valor-Segura et al., 2010).

Por otro lado, la tristeza y su expresión psicopatológica, la depresión, se asocian fuertemente a la violencia. Se considera a la tristeza una emoción complementaria al miedo ya que constituye buena parte de la respuesta de la persona agredida. La depresión sitúa a la víctima en una posición de indefensión y fuente de irritación y frustración para el agresor (Niedenthal et al., 2006). Por otro lado, los estudios muestran que hay una menor aceptación de la mujer si evidencia conductas agresivas y es más probable que contengan su ira, mientras que es más probable que los hombres la manifiesten (Etxebarria et al., 2009). La ira del hombre predecía la violencia, aunque no ocurría lo mismo con la ira femenina - recordemos que la violencia recíproca era un factor de riesgo de ser víctima y podemos pensar que en parte se asocia a la ira de esta última.

La culpa y la vergüenza se han asociado a la violencia, emociones definidas como sociales y morales, motivadoras y controladoras de la conducta. Paradójicamente, los estudios muestran que las victimas sienten vergüenza por ser humilladas y culpabilidad por las interacciones negativas. La vergüenza genera un estado emocional desagradable e interrumpe la acción, al tiempo que genera una cierta confusión mental en la víctima. Por otro lado, la culpa tiene por referente una acción concreta y promueve la necesidad de reparación, mientras que la vergüenza es más generalizada y más orientada hacia uno mismo (Sheikh y Janoff-Bulman, 2010). Para poder librarse de estas emociones, la víctima recurre a estrategias como la reinterpretación cognitiva, la negación y el olvido de la situación, como en la atribución benévola de las causas de la agresión y el perdón de ésta. Estas emociones de culpa y vergüenza, junto con las de amor, miedo y soledad, que se generan en la víctima en la interacción con el maltratador, además de las estrategias de control ejercidas

\section{Referencias}

Abramsky, T., Watts, C. H., Garcia-Moreno, C., Devries, K., Kiss, L., Ellsberg, M., Ellsberg, M., Jasen, H. y Heise, L. (2011). What factors are associated with recent intimate partner violence? findings from the WHO multi-country study on women's health and domestic violence. BMC Public Health, 11 (1), 1471-2458. doi:10.1186/1471-2458-11-109

Adelman, M. (2003). The military, militarism, and the militarization of domestic violence. Violence against Women, 9, 1118-1152. doi: $10.1177 / 1077801203255292$.

Ali, T. S., Asad, N., Mogren, I. y Krantz, G. (2011). Intimate partner violence in urban Pakistan: prevalence, frequency, and risk factors. International Journal of Women's Health, 3, 105-115. doi: 10.2147/IJWH.S17016 por éste, provocan que se prolongue la situación de maltrato a la que es sometida.

Por otra parte, las actitudes a favor de la violencia la refuerzan. La justificación de la violencia, la adaptación funcional, la tolerancia a la agresión y la empatía hacia el agresor se han definido como los elementos básicos de ésta (Martín, 1999). Las mujeres que más justifican la violencia tienen mayor riesgo de sufrirla al mismo tiempo que se mantienen más tiempo dentro de la relación traumática. La mujer tiende a centrarse en los aspectos positivos de su pareja, a negar o minimizar el problema y a auto-engañarse, llegando a normalizar, tolerar y justificar su situación de violencia (Echeburúa et al., 2002). La adaptación paradójica o síndrome de Estocolmo (Alonso, Manso y Sánchez, 2010) y la dependencia emocional de la víctima sobre el maltratador (Echeburúa et al., 2002) pueden explicar que la mujer se mantenga durante más tiempo en la relación.

La limitación más importante de esta revisión es que la predicción del riesgo incluye otro tipo de mediciones referidas a la naturaleza y la gravedad o intensidad de los efectos que produce en las víctimas. En este estudio se tienen en cuenta todos los que por sí solos han sido asociados con un incremento sobre el riesgo de violencia, independientemente del tipo de abuso que se haya recibido. Por este motivo es posible que se encuentren amplias diferencias entre los estudios, en cuanto a los porcentajes de violencia expuestos, ya que éstos dependen de cómo se defina la violencia, del método utilizado para medirla, del tipo de población estudiada (población general, población clínica, etc.) y del periodo de tiempo establecido para la pregunta (por ejemplo, un año o todo el período vital). Además para algunos factores de riesgo no se han encontrado estudios cuantitativos que aporten datos estadísticos concluyentes y por tanto deben de ser tomados con cautela (religión, globalización o duración de la relación). Otro problema es que no todas estas variables han sido estudiadas como causa primaria, incremento o forma de perpetuación de la violencia en la pareja, sino que hay otras revisiones que las han considerado como consecuencia de la situación de maltrato, sobre todo cuando lo que se analizan son las repercusiones sobre la salud de las víctimas primarias.

Notas y/o Agradecimientos.- Esta investigación se sustenta en la siguiente beca: PIF/UPV/12/226 para la formación del personal investigador.

Alonso, M. B., Manso, J. M. M. y Sánchez, M. E. G. B. (2010). Revisión teórica del maltrato psicológico en la violencia conyugal. Psicología y salud, $20(1), 65-75$

Andersson, N., Cockcroft, A., Ansari, N., Omer, K., Chaudhry, U. U., Khan, A. y Pearson, L. (2009). Collecting Reliable Information About Violence Against Women Safely in Household Interviews Experience From a Large-Scale National Survey in South Asia. Violence Against Women, 15 (4), 482-496. doi: 10.1177/1077801208331063

Archer, J. (2000). Sex differences in Aggression between heterosexual partners: a Meta-Analitytic review. Psychological Bulletin, 126 (5), 651-680. doi: 10.1037/0033-2909.126.5.651 . 
Archer, J. (2006). Cross-cultural differences in physical aggression between partners: A social-role analysis. Personality and Social Psychology Review, 10, 113-133. doi: 10.1207/s15327957pspr1002_3.

Arzu R. y Pinky S. R. (2001). A study on the psycho-social impacts of violence against women and girls with special focus on rape, incest and polygamy. Nepal: Saathi and SNV.

Beydoun, H. A., Beydoun, M. A., Kaufman, J. S., Lo, B. y Zonderman, A. B. (2012). Intimate partner violence against adult women and its association with major depressive disorder, depressive symptoms and postpartum depression: a systematic review and meta-analysis. Social Science \& Medicine, 75(6), 959-975.

Cáceres, J. (2004). Violencia Física, Psicológica y sexual en el Ámbito de la pareja: Papel del Contexto. Clínica y salud, 15 (1), 33-34.

Cáceres, A. y Cáceres, J. (2006). Violencia en relaciones íntimas en dos etapas evolutivas. Intenational Journal of Clinical and Health Psychology, 6 (2), 271-284.

Campbell J., Garcia-Moreno, C. y Sharps, P. (2004). Abuse during pregnancy in industrialized and developing countries. Violence Against Women, 10 (7), 770-789. doi: 10.1177/1524838005277441.

Capaldi, D. M., Shortt, J. W. y Kim, H. K. (2005). A life span developmental system perspective on aggression toward a partner. En W. M. Pinsof y J. Lebow (Eds.), Family Psychology: the Art of the Science (pp. 141-167). Oxford, United Kingdom: Oxford Press. ISBN: 0195135571.

Carboné-López, K. y Kruttschnitt, C. (2010). Risky relationships? Assortative mating and women's experiences of intimate partner violence. Crime \& Delinquency, 56 (3), 358-384. doi:10.1177/0011128709333727.

Catani, C., Schauer, E. y Neuner, F. (2008). Beyond individual war trauma: Domestic violence against children in Afghanistan and Sri Lanka. Journal of Marital and Family Therapy, 34 (2), 165-176. doi: 10.1111/j.17520606.2008.00062.x.

Chan, K. L., Tiwari, A., Fong, D. Y., Leung, W. C., Brownridge, D. A. y Ho, P. C. (2011). Correlates of in-law conflict and intimate partner violence against Chinese pregnant women in Hong Kong. Journal of interpersonal violence, 24 (1), 97-110. doi:10.1177/0886260508315780.

Chen, P. H. y White, H. R. (2004). Gender Differences in Adolescent and Young Adult Predictors of Later Intimate Partner Violence A Prospective Study. Violence Against Women, 10 (11), 1283-1301. doi: $10.1177 / 1077801204269000$

Clark, C. J., Everson-Rose, S. A., Suglia, S. F., Btoush, R., Alonso, A. y HajYahia, M. M. (2010). Association between exposure to political violence and intimate-partner violence in the occupied Palestinian territory: a cross-sectional study. The Lancet, 375 (9711), 310-316.

Cramer, D. (2004). Satisfaction with a romantic relationship, depression, support and conflict. Psychology and Psychotherapy: Theory, Research and Practice, 77 (4), 449-461. doi:10.1348/1476083042555389

Dollard, J., Doob, L., Miller, N., Mowrer, O. y Sears, R. (1939). Frustration and Aggression. New Haven, $\mathrm{CN}$ : Yale University Press.

Dutton, D. G. (1995). The domestic assault of women: Psychological and criminal justice perspectives. Vancouver, BC: UBC Press.

Dutton, D. (2006). Rethinking domestic violence. Vancouver, BC, Canada: UBC Press. ISBN: 978-0-7748-1304-4.

Echeburúa, E., Amor, P.J. y De Corral, P. (2002). Mujeres maltratadas en convivencia prolongada con el agresor: variables relevantes. Acción Psicológica, 2, 135-150.

Echeburúa, E., Sarasua, B., Zubizarreta, I., Amor, P. J. y Corral, P. (2010). Variables predictoras del rechazo, abandono y fracaso terapéutico en hombres violentos contra su pareja tratados psicológicamente en un marco comunitario. International Journal of Clinical and Health Psychology, 10 (3), 403-420.

Etxebarria, I., Ortiz, M. J., Conejero, S. y Pascual, A. (2009). Intensity of habitual guilt in men and women: Differences in interpersonal sensitivity and the tendency towards anxious-aggressive guilt. The Spanish Journal of Psychology, 12 (2), 540-554.

Expósito, F., Herrera, M. C., Moya, M. y Glick, P. (2010). Don't Rock the Boat: Women's Benevolent Sexism Predicts Fears of Marital Violence. Psychology of Women Quarterly, 34 (1), 36-42.

Femat, M.L. (2008).La función del miedo en la violencia de pareja. Tramas, 30, 191-212.
Fincham, F. D., Beach, S. R. H. y Davila, J. (2007). Longitudinal relations between forgiveness and conflict resolution in marriage. Journal of Family Psychology, 21, 542-545. doi:10.1037/0893-3200.21.3.542.

Folkman, S. (ed.). (2010). The Oxford handbook of stress, health, and coping. Oxford University Press. ISBN 978-94-007-4275-8

García-Moreno, C., Jansen, H., Ellsberg, M., Heise, L. y Watts, C. H. (2006). Prevalence of intimate partner violence: findings from the WHO multicountry study on women's health and domestic violence. The Lancet, 368 (9543), 1260- 1269. doi:10.1016/S0140- 6736(06)69782-1

Gordon, K. C., Burton, S. y Porter, L. (2004). Predicting the intentions of women in domestic violence shelters to return to partners: Does forgiveness play a role? Journal of Family Psychology, 18, 331-338. doi:10.1037/0893-3200.18.2.331

Graña, J. L., Rodríguez, M. J. y Peña, M. E. (2009). Agresión hacia la pareja en una muestra de la Comunidad de Madrid: Análisis por género. Psicopatología clínica, legal y forense, 9,7-28.

Hazen, A. L. y Soriano, F. I. (2007). Experiences with intimate partner violence among Latina women. Violence Against Women, 13 (6), 562-582. doi: $10.1177 / 1077801207301558$

Hedin, L. W. (2000). Postpartum, also a risk period for domestic violence. European Journal of Obstetrics, Gynecology, and Reproductive Biology, 89 (1), 4145. doi: 10.1016/S0301-2115(99)00164-5

Hotaling, G. T. y Sugarman, D. B. (1986). An analysis of risk markers in husband to wife violence: The current state of knowledge. Violence and victims, 1 (2), 101-124.

Instituto de la Mujer (2007). Denuncias por malos tratos producidos por la pareja o expareja según grupos de edad. Año 2002-2007. Instituto de la Mujer. Recuperado de: http://www.mtas.es/mujer/mujeres/cifras/violencia/denuncias_tablas .htm

Jewkes, R. (2002). Intimate partner violence: causes and prevention. Lancet, 359 (9315), 1423-1429. doi: 10.1016/S0140-6736(02)08357-5

Karney, B. R. y Bradbury, T. N. (2000). Attributions in marriage: State or trait? A growth curve analysis. Journal of Personality and Social Psychology, 78, 295-309. doi:10.1037/0022 3514.78.2.295

Kaya, Y. y Cook, K. J. (2010). A cross-national analysis of physical intimate partner violence against women. International Journal of Comparative Sociology, 51 (6), 423-444. Doi. 10.1177/0020715210386155.

Kim, J., Talbot, N. L. y Cicchetti, D. (2009). Childhood abuse and current interpersonal conflict: The role of shame. Child Abuse \& Neglect, 33(6), 362-371. doi:10.1016/j.chiabu.2008.10.003

Kishor, S. y Johnson, K. (2004). Profiling domestic violence-a multi-country study. Calverton, Maryland: ORC Macro.

Kliewer, W. y Zaharakis, N. (2013). Community violence exposure, coping, and problematic alcohol and drug use among urban, female caregivers: A prospective study. Personality and Individual Differences. 55 (4), 361366. doi:10.1016/j.paid.2013.03.020

Lawoko, S., Dalal, K., Jiayou, L. y Jansson, B. (2007) Social inequalities in intimate partner violence: A study of women in Kenya. Violence and Victims, 22 (6), 773-784. doi: 10.1891/088667007782793101

López-Zafra, E. y Rodríguez, N. R. (2008). Relación entre cultura del honor, celos y Satisfacción en la pareja. Boletín de Psicología, 94, 7-22.

Lucas, T., Parkhill, M. R., Wendorf, C. A., Imamoglu, E. O., Weisfeld, C. C., Weisfeld, G. E. y Shen, J. (2008). Cultural and Evolutionary Components of Marital Satisfaction A Multidimensional Assessment of Measurement Invariance. Journal of cross-cultural psychology, 39 (1), 109-123. doi: $10.1177 / 0022022107311969$.

Martín, F. M. (1999). La violencia en la pareja. Pan American Journal Of Public Health, 5, 245-258.

Martín-Baró, I. (1983). Acción e ideología. Psicología social desde Centroamérica. San Salvador: UCA Editores.

McCollum, E. E. y Stith, S. M. (2008). Couples treatment for interpersonal violence: a review of outcome research literature and current clinical practices. Violence and Victims, 23 (2), 187-201. doi:10.1891/08866708.23.2.187

McNulty, J. K. y Fincham, F. D. (2012). Beyond positive psychology? Toward a contextual view of psychological processes and well-being. American Psychologist, 67 (2), 101. doi: 10.1037/a0024572

McNulty, J. K. y Russell, V. M. (2010). When "negative" behaviors are positive: A contextual analysis of the long-term effects of problemsolving 
behaviors on changes in relationship satisfaction. Journal of Personality and Social Psychology, 98, 587-604. doi:10.1037/ a0017479.

McNulty, J. K., O’Mara, E. M. y Karney, B. R. (2008). Benevolent cognitions as a strategy of relationship maintenance: "Don't sweat the small stuff' but it's not all small stuff. Journal of Personality and Social Psychology, 94, 631-646. doi:10.1037/0022-3514.94.4.631.

Moral, J., Rosales, F. L., Loving, R. D. y Martínez, Y. I. C. (2011). Diferencias de género en afrontamiento y violencia en la pareja. Revista CES Piscología, 4 (2), 29-46.

Moya, M., Páez, D., Glick, P., Fernández, I. y Poeschl, G. (2002). Masculinidad-feminidad y factores culturales. Revista Española de y Motivación y Emoción, 3, 127-142.

Nasir, K. y Hyder, A. A. (2003). Violence against pregnant women in developing countries: Review of evidence. European Journal of Public Health, 13 (2), 105-107. doi: 10.1093/eurpub/13.2.105

Naved, R. T., Azim, S., Bhuiya, A. y Persson, L. A. (2006). Physical violence by husbands: magnitude, disclosure and help-seeking behavior of women in Bangladesh. Social Science \& Medicine, 62 (12), 2917-2929. doi:10.1016/j.socscimed.2005.12.001

Nayak, M. B., Byrne, C. A., Martin, M. K. y Abraham, A. G. (2003). Attitudes toward violence against women: A cross-nation study. Sex Roles, 49(7-8), 333-342

Niedenthal, P. M., Krauth-Gruber, S. y Ric, F. (2006). Psychology of emotion: Interpersonal, experiential, and cognitive approaches. Psychology Press New York.

Nocentini, A., Menesini, E. y Pastorelli, C. (2010). Physical Dating Aggression growth during adolescence. Journal of Abnormal Child Psychology, 38, 353-365.

O'Leary, K. D. (1999). Developmental and affective issues in assessing and treating partner aggression. Clinical Psychology: Science and Practice, 6, 400414.

Organización Mundial de la Salud (2005). Estudio multipaís de la OMS sobre salud de la mujer y violencia doméstica. Suiza: OMS.

Páez, D. y Ubillos, S. (2004). Agresión. En D. Páez, I. Fernández, S. Ubillos y E. Zubieta (Coords), Psicología social, cultura y educación (pp. 553-604). Madrid: Pearson/Prentice Hall.

Páez, D., Martínez Sánchez, F., Sevillano Triguero, V., Mendiburo Seguel, A. y Campos, M. (2012) Medidas de estilos de regulación afectiva (MARS) ampliado en ira y tristeza. Psicothema, 24, 249-254

Pérez, V. A., Bosch, E., Navarro, C. y Ramis, C. (2006). Las creencias y actitudes sobre la violencia contra las mujeres en la pareja: Determinantes sociodemográficos, familiares y formativos. Anales de Psicología, 22 (2), 251-259.

Pérez, J. A., Páez, D, Navarro, E. y Arias, A. (2001).Conflicto de mentalidades: cultura del honor frente a liberación de la mujer. Revista Electrónica de Motivación y Emoción, 4 (8-9), 1-23.

Pueyo, A., y Redondo, S. (2007). Predicción de la violencia: entre la peligrosidad y la valoración del riesgo de violencia. Papeles del Psicólogo, 28 (3), 157-173.

Radford, L. y Tsutsumi K (2004). Globalization and violence against women: Inequalities in risks, responsibilities and blame in the UK and Japan. Women's Studies International Forum, 27, 1-12. doi:10.1016/j.wsif.2003.12.008

Riggs, D. S., Caulfield, M. B y Street, A. E. (2000). Risk for domestic violence: factors associated with perpetration and victimization. Journal of Clinical Psychology, 56 (10), 1289-1316. doi:10.1002/1097-4679

Ruiz-Pérez, I., Mata-Pariente, N. y Plazaola-Castaño, J. (2006). Women's response to intimate partner violence. Journal of Interpersonal Violence, 21 (9), 1156-1168. doi: 10.1177/0886260506290421

Saile, R., Neuner, F., Ertl, V. y Catani, C. (2013). Prevalence and predictors of partner violence against women in the aftermath of war: A survey among couples in Northern Uganda. Social Science \& Medicine, 86, 17-25. doi: 10.1016/j.socscimed.2013.02.046

Schumacher, J. A., Feldbau-Kohn, S., Smith Slep, A. M. y Heyman, R. E. (2001). Risk factors for male-to-female partner physical abuse. Aggression and Violent Behavior, 6 (2), 281-352. doi: 10.1016/S1359-1789(00)000276.
Schuler, S. R., Hashemi, S. M., Riley, A. P. y Akhter, S. (1996). Credit programs, patriarchy and men's violence against women in rural Bangladesh. Social XDcience \& Medicine, 43(12), 1729-1742.

Sheikh, S. y Janoff-Bulman, R. (2010). Tracing the self-regulatory bases of moral emotions. Emotion Review, 2, 386-396. doi: 10.1177/1754073910374660

Stewart, F. y Brown, G. (2010). Fragile states. Oxford, UK: University of Oxford, Center for Research on Inequality, Human Security and Ethnicity (CRISE).

Stith, S. M., Green, N. M., Smith, D. B. y Ward, D. B. (2008). Marital satisfaction and marital discord as risk markers for intimate partner violence: A meta-analytic review. Journal of Family Violence, 23(3), 149-160. doi: 10.1007/s10896-007-9137-4

Stith, S. M., Rosen, K. H., Middleton, K. A., Busch, A. L., Lundeberg, K. y Carlton, R. P. (2000). The intergenerational transmission of spouse abuse: A meta-analysis. Journal of Marriage and the Family, 62, 640-654. doi: 10.1111/j.1741-3737.2000.00640.x

Stith, S. M., Smith, D. B., Penn, C. E., Ward, D. B. y Tritt, D. (2004). Intimate partner physical abuse perpetration and victimization risk factors: A meta-analytic review. Aggression and violent behavior, 10 (1), 65-98. doi:10.1016/j.avb.2003.09.001.

Suárez, E. y Gadalla, T. M. (2010). Stop blaming the victim: A meta-analysis on rape myths. Journal of Interpersonal Violence, 25(11), 2010-2035.

Sugarman, D. B. y Hotaling, G. T. (1997). Intimate Violence and Social Desirability A Meta-Analytic Review. Journal of Interpersonal Violence, 12(2), 275-290. doi: 10.1177/088626097012002008

Sugarman, D. B. y Frankel, S. L. (1996). Patriarchal ideology and wifeassault: A meta-analytic review. Journal of Family Violence, 11(1), 13-40.

Sullivan, C. M. (2010). Victim services for domestic violence. In M. P. Koss, J. W. White, \& A. E. Kazdin (Eds.), Violence against women and children: Navigating solutions (pp. 183-197). Washington, DC: American Psychological Association.

Timmons, P. A. y O’Leary, K. D. (2004). Physical and Psychological Partner Aggression Across a Decade: A Growth Curve Analysis. Violence and Victims, 19 (1), 3-16. . doi:10.1891/vivi.19.1.3.33236

Tokuç, B., Ekuklu, G. y Avcioğlu, S. (2010). Domestic Violence against married women in Edirne. Journal of Interpersonal Violence, 25 (5), 832-847. doi: $10.1177 / 0886260509336960$

Ubillos, S., Martín-Beristain, M., Garaigordobil, M. y Halperín, E. (2011). Agresión, odio, conflictos intergrupales y violencia colectiva. En D. Páez, C. Martín-Beristain, J.L. González, N. Basabe y J. de Rivera (Eds.), Superando la violencia colectiva y construyendo cultura de paz. (pp. 15-37). Madrid: Fundamentos. Colección Ciencia. ISBN: 978-84-245-1236-1.

Uthman, O. A., Lawoko, S. y Moradi, T. (2010). Sex disparities in attitudes towards intimate partner violence against women in sub-Saharan Africa: a socio-ecological analysis. BMC Public Health, 10(1), 223.)

Valor-Segura, I., Expósito, F. y Moya, M. (2010). Emociones poderosas y no poderosas ante conflictos de pareja: diferencias de género. Intervención Psicosocial, 19 (2), 129-134. doi: 10.5093/in2010v19n2a4

Villarreal, A. (2007). Women's employment status, coercive control, and intimate partner violence in Mexico. Journal of Marriage and Family 69, 418434. doi: 10.1111/j.1741-3737.2007.00374.x

Vives-Cases, C., Álvarez-Dardet, C., Gil-González, D., TorrubianoDomínguez, J., Rohlfs, I. y Escribà-Agüir, V. (2009). Perfil sociodemográfico de las mujeres afectadas por violencia del compañero íntimo en España. Gaceta Sanitaria, 23 (5), 410-414. doi: 10.1590/S0213 91112009000500009

Weldon, S.L. (2002). Protest, Policy, and the Problem of Violence Against Women: A Cross-national Comparison. Pittsburgh, PA: University of Pittsburgh Press. ISSN: 0020-7152

Wendt, S. (2008). Christianity and domestic violence: Feminist poststructuralist perspectives. Journal of Women and Social Work, 23 (2), 144-155. doi: $10.1177 / 0886109908314326$

West, T. (1999). Wounds of the Spirit: Black Women, Violence, and Resistance Ethics. New York: New York. University Press

(Artículo recibido: 20-12-2013; revisado: 04-01-2015; aceptado: 18-01-2015) 